\title{
EVN and global VLBI observations of candidate radio sources for alignment between the ICRF and the future Gaia frame
}

\author{
G. Bourda*, A. Collioud, P. Charlot \\ Univ. Bordeaux, LAB, UMR 5804, F-33270, Floirac, France \\ CNRS, LAB, UMR 5804, F-33270, Floirac, France \\ E-mail: bourda@obs.u-bordeauxl.fr
}

\section{R. Porcas}

MPIfR Bonn, Germany

\section{S. Garrington}

Jodrell Bank Observatory, The University of Manchester, UK

The space astrometry mission Gaia will construct a dense optical QSO-based celestial reference frame. For consistency between optical and radio positions, it will be important to align the Gaia and VLBI frames with the highest accuracy. However, the number of quasars that are bright in optical wavelength (for the best position accuracy with Gaia), that have a compact core (to be detectable on VLBI scales), and that do not exhibit complex structures (to ensure a good astrometric quality), was found to be limited. It was hence realized that the densification of the list of such objects was necessary. Accordingly, we initiated a multi-step VLBI observational project, dedicated to finding additional suitable radio sources for aligning the two frames. The sample consists of $\sim 450$ optically-bright weak extragalactic radio sources, which have been selected by cross-correlating optical and radio catalogs. The initial observations, aimed at checking whether these sources are detectable with VLBI, and conducted with the European VLBI Network (EVN) in 2007, showed an excellent $\sim 90 \%$ detection rate. The second step, dedicated to identify the most point-like sources of the sample, by imaging their VLBI structures, was initiated in 2008. About $25 \%$ of the detected targets were observed with the Global VLBI array (EVN+VLBA; Very Long Baseline Array) during a pilot imaging experiment, revealing that about $50 \%$ of them are point-like sources on VLBI scales. The rest of the sources were observed during three additional imaging experiments in March 2010, November 2010 and March 2011. The final stage was initiated in June 2012 with a first global observing experiment, dedicated to measuring accurately the VLBI position of the most point-like of such sources. In this paper, we present the results of the imaging campaigns and draw plans for the future.

11th European VLBI Network Symposium \& Users Meeting,

October 9-12, 2012

Bordeaux, France

${ }^{*}$ Speaker. 


\section{Context}

During the past decade, the IAU (International Astronomical Union) fundamental celestial reference frame was the ICRF (International Celestial Reference Frame; [1, 2]), composed of the VLBI (Very Long Baseline Interferometry) positions of 717 extragalactic radio sources, measured using dual-frequency S/X observations (2.3 and $8.4 \mathrm{GHz}$ ). Since 1 January 2010, the IAU fundamental celestial reference frame has been the ICRF2 [3], successor of the ICRF. It includes VLBI coordinates for 3414 extragalactic radio sources, with a floor in position accuracy of $60 \mu$ as and an axis stability of $10 \mu$ as.

The European space astrometry mission Gaia, to be launched by the end of 2013, will survey all stars and QSOs (Quasi Stellar Objects) brighter than apparent optical magnitude 20 [4]. Using Gaia, optical positions will be determined with an unprecedented accuracy, ranging from a few tens of $\mu$ as at magnitude 15-18 to about $200 \mu$ as at magnitude 20 [5]. Unlike Hipparcos, Gaia will permit the realization of the extragalactic celestial reference frame directly at optical bands, based on the QSOs that have the most accurate positions. A preliminary Gaia catalog is expected to be available by 2015 with the final version released by 2020 .

In this context, aligning VLBI and Gaia frames will be crucial for ensuring consistency between the measured radio and optical positions. This alignment, to be determined with the highest accuracy, requires several hundreds of common sources, with a uniform sky coverage and very accurate radio and optical positions. Obtaining such accurate positions implies that the link sources must be brighter than optical magnitude 18 [6], and must not show extended VLBI structures.

In a previous study, we investigated the potential of the ICRF for this alignment and found that only 70 sources ( $10 \%$ of the catalog) were appropriate for this purpose [7]. This highlighted the need to identify additional suitable radio sources, which was the goal of a VLBI program that we initiated five years ago. This program has been devised to observe 447 optically-bright extragalactic radio sources, on average 20 times weaker than the ICRF sources, extracted from the NRAO VLA Sky Survey, a dense catalog of weak radio sources [8]. The observing strategy to detect, image, and measure accurate VLBI positions for these sources is described in [9].

In this paper, we give the status of this program, by focussing on the latest imaging experiments, and outline future prospects.

\section{The observing program}

\section{VLBI detection}

The initial observations, whose goal was to assess the VLBI detectability of the 447 targets, were conducted with the European VLBI Network (EVN), recording at 1 Gbps in a geodetic-style dualfrequency S/X mode, in June and October 2007 (during two 48-hours experiments, EC025A and EC025B, respectively). These showed excellent detection rates of $97 \%$ at X-band and $89 \%$ at Sband. Overall, 398 sources were detected at both frequencies, corresponding to an overall detection rate of about $89 \%$ [9].

\section{VLBI imaging}

Proceeding further with our program, the second step was targeted at imaging the sources previ- 
ously detected, using the global VLBI network (EVN+VLBA; Very Long Baseline Array), recording at $512 \mathrm{Mbps}$ in a dual-frequency S/X mode, in order to identify the most point-like sources and therefore the most suitable ones for the alignment.

In total, four global VLBI imaging campaigns were carried out, during 192 hours, to observe 395 sources ( 3 of the 398 detected were gravitational lenses). Table 1 summarizes these experiments and their corresponding results [10,11]. In total, X-band VLBI maps were determined for 250 sources (i.e. $63 \%$ successful mapping; see Fig. 1 for some examples). The total flux densities of these sources were determined at both S- and X-bands from these maps (see Fig. 2). Figure 3 shows that about $50 \%$ of the targets (i.e. 119 sources) we could image are point-like sources (i.e. $\mathrm{X}$-band continuous structure index $<3.0$; see definition in e.g. [3]).

\section{VLBI astrometry}

The final stage of this program, dedicated to determining very accurate VLBI positions (i.e. position accuracy wanted to better than 200-300 $\mu$ as) for the most point-like sources of the sample (i.e. 119 sources) began with 3 days of Global VLBI observations in June 2012.

\begin{tabular}{lllrrr}
\hline \hline Experiment & Date & $\begin{array}{l}\text { Duration } \\
(\mathrm{hrs})\end{array}$ & $\begin{array}{r}\text { Number of } \\
\text { sources } \\
\text { observed }\end{array}$ & $\begin{array}{r}\text { Number of } \\
\text { sources } \\
\text { imaged (\%) }\end{array}$ & $\begin{array}{r}\text { Number of } \\
\text { point-like } \\
\text { sources (\%) }\end{array}$ \\
\hline GC030 & March 2008 & 48 & 105 & $105(100 \%)$ & $47(45 \%)$ \\
GC034A & March 2010 & 48 & 97 & $63(65 \%)$ & $32(51 \%)$ \\
GC034BCD & November 2010 & 58 & 118 & $52(44 \%)$ & $26(50 \%)$ \\
GC034EF & March 2011 & 38 & 75 & $30(40 \%)$ & $14(47 \%)$ \\
\hline
\end{tabular}

Table 1: Summary of the four global VLBI imaging experiments, in terms of observations and results.

\section{Summary and future prospects}

Within the next few years, the alignment between optical and radio frames will benefit from this multi-step VLBI project. Obtaining such an alignment with the highest accuracy is essential, not only to ensure consistency between measured radio and optical positions, but also to measure directly core shifts within AGNs. This will be of high interest in the future for probing AGN jets properties. Furthermore, while making the Gaia link possible, these new VLBI positions will also serve in the future to densify the VLBI frame at the same time.

\section{Acknowledgements}

The authors would like to thank the VLBI friends at the EVN and VLBA observing stations. This work has benefited from research funding from the European Community's sixth Framework Programme under RadioNet R113CT 2003 5058187. This work has been supported by the European Community Framework Programme 7, Advanced Radio Astronomy in Europe, grant agreement nl.: 227290 and nl.: 283393. The EVN is a joint facility of European, Chinese, South African 

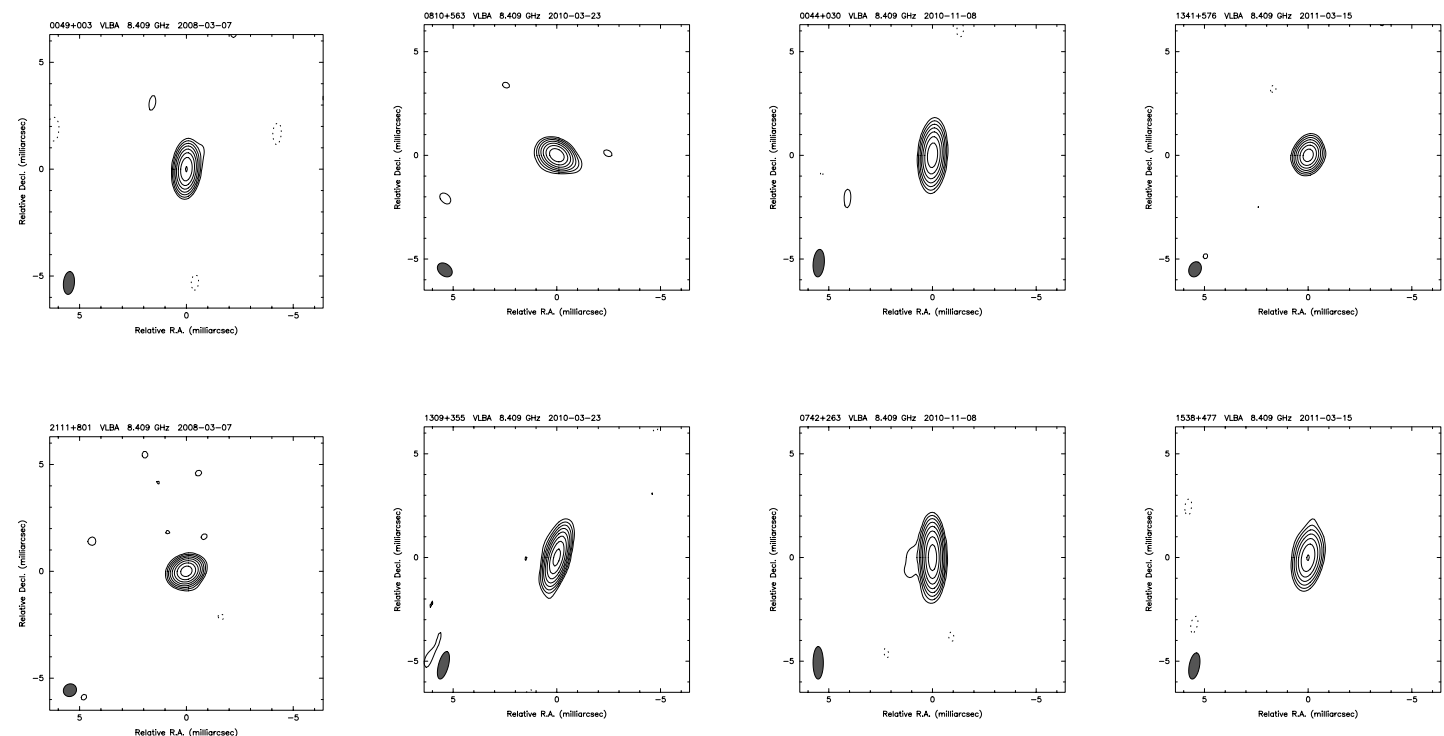

Figure 1: Examples of VLBI maps (X-band; first contour level at 1-4\%), determined during the four VLBI imaging experiments, for sources considered as point-like (i.e. with a good astrometric quality). From left to right: $0049+003$ and $2111+801$ (GC030), 0810+563 and 1309+355 (GC034A), 0044+030 and $0742+263$ (GC034BCD), 1341+576 and 1538+477 (GC034EF).

and other radio astronomy institutes funded by their national research councils. The VLBA is part of the National Radio Astronomy Observatory (NRAO), which is operated by Associated Universities, Inc., under cooperative agreement with the National Science Foundation.

\section{References}

[1] C. Ma, E.F. Arias, T. Eubanks, A.L. Fey, A.-M. Gontier, et al., 1998, The International Celestial Reference Frame as Realized by Very Long Baseline Interferometry, AJ 116, 516-546.

[2] A.L. Fey, C. Ma, E.F. Arias, P. Charlot, M. Feissel-Vernier, et al., 2004, The Second Extension of the International Celestial Reference Frame: ICRF-EXT.1, AJ 127, 3587-3608.

[3] IERS Technical Note 35, 2009, The Second Realization of the International Celestial Reference Frame by Very Long Baseline Interferometry, Presented on behalf of the IERS/IVS Working Group, A. Fey, D. Gordon \& C. Jacobs (eds.), Frankfurt am Main: Verlag des Bundesamts für Kartographie und Geodäsie, ISBN 3-89888-918-6, 204 p.

[4] M.A.C. Perryman, K.S. de Boer, G. Gilmore, E. Høg, M.G. Lattanzi, et al., 2001, GAIA: Composition, formation and evolution of the Galaxy, A\&A 369, 339-363.

[5] L. Lindegren, C. Babusiaux, C. Bailer-Jones, U Bastian, A.G.A. Brown, et al., 2008, The Gaia mission: science, organization and present status, In: Proceedings of IAU Symposium 248, A Giant Step: from Milli- to Micro-arcsecond Astrometry, W. Wenjin, I. Platais \& M. Perryman (eds), Cambridge University Press, 217-223. 
[6] F. Mignard, 2003, Future Space-Based Celestial Reference Frame, In: Proceedings of IAU General Assembly XXV, Joint Discussion 16: The International Celestial Reference System: Maintenance and Future Realization, R. Gaume, D. McCarthy \& J. Souchay (eds.), 133-140.

[7] G. Bourda, P. Charlot and J.-F. Le Campion, 2008, Astrometric suitability of optically-bright ICRF sources for the alignment with the future Gaia celestial reference frame, A\&A 490, 403-408.

[8] J.J. Condon, W.D. Cotton, E.W. Greisen, Q.F. Yin, R.A. Perley, et al., 1998, The NRAO VLA Sky Survey, AJ 115, 1693-1716.

[9] G. Bourda, P. Charlot, R. Porcas and S. Garrington, 2010, VLBI observations of optically-bright extragalactic radio sources for the alignment of the radio frame with the future Gaia frame: I-Source detection, A\&A 520, A113.

[10] G. Bourda, A. Collioud, P. Charlot, R. Porcas and S. Garrington, 2011, VLBI observations of optically-bright extragalactic radio sources for the alignment of the radio frame with the future Gaia frame: II-Imaging candidate sources, A\&A 526, A102.

[11] G. Bourda, A. Collioud, P. Charlot, R. Porcas and S. Garrington, 2013, VLBI observations of optically-bright extragalactic radio sources for the alignment of the radio frame with the future Gaia frame: III-Final Images of candidate sources, A\&A (in prep.) 

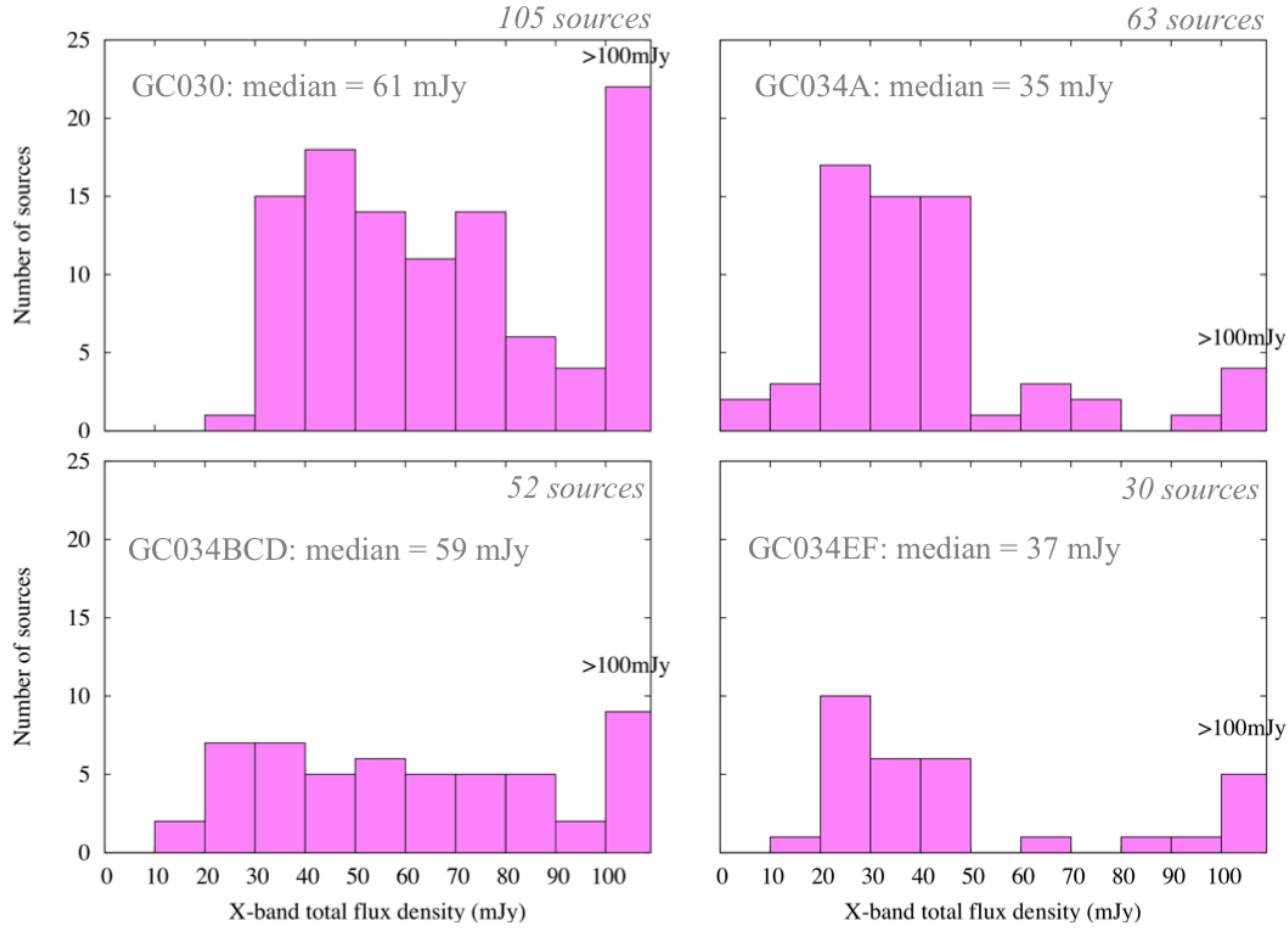

Figure 2: Distribution of the X-band total flux density for the 250 sources detected during the four VLBI imaging experiments (units in $\mathrm{mJy}$ ). The corresponding median values are given for each plot.
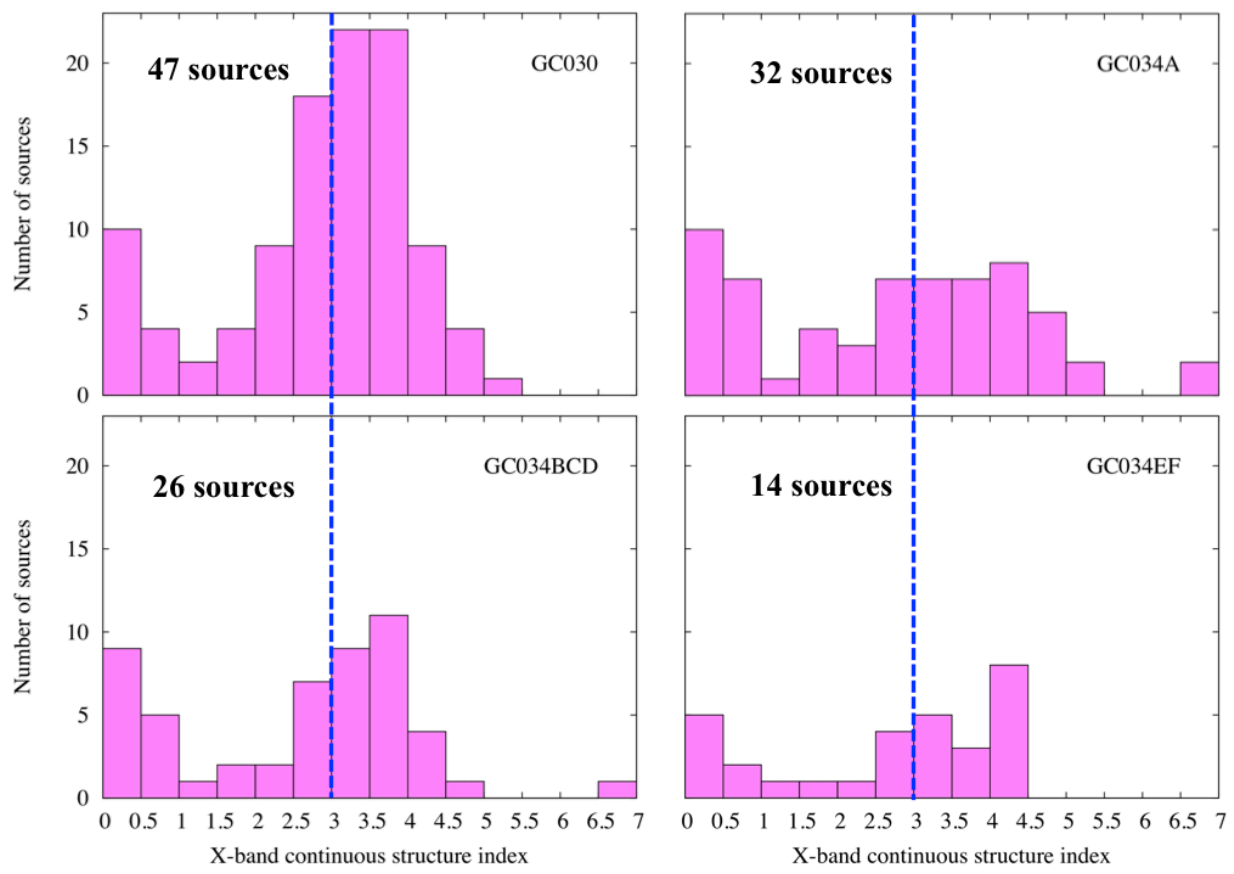

Figure 3: Distribution of the $\mathrm{X}$-band continuous structure index for the 250 sources detected during the four VLBI imaging experiments. The number of sources considered as point-like (i.e. X-band continuous structure index $<3.0$ ) is given (in bold) for each plot. 